\title{
The road to E. faecium pathogenesis
}

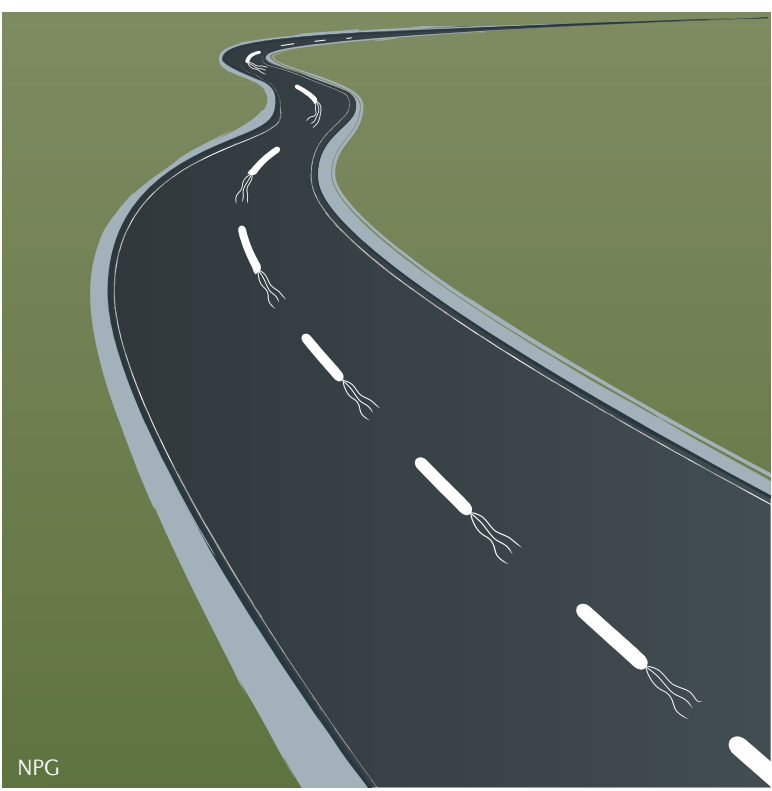

access to a new source of carbon and energy is a major driver of E. faecium evolution.
Although Enterococcus faecium was originally a gut commensal organism, it emerged as a leading cause of hospital-acquired infections in the 1980s. Here, Gilmore and colleagues use a genome analysis-based approach to obtain insights into the the genomes of 51 strains that belong to different multilocus sequence types and were isolated from different environments over the past 60 years. They then used this information to construct a phylogenetic tree based on SNPs. This revealed that strains causing most hospital-acquired infections stem from a hypermutating lineage (clade A1) that branched off a lineage which includes animal commensals (clade A2). The lineage that includes human commensals (clade B) was found to be more distantly related.

Next, the authors assessed interclade differences in gene content that might explain their distinct lifestyles. Clade A1 strains had a larger overall genome size than A2 and $B$ strains, indicative of the acquisition of new functions. Notably, clade A1 contained higher levels of mobile genetic elements, including plasmids, phages and other genomic islands, than clades A2 and B. These mobile elements were associated with a higher number of genome rearrangements (such as inversions)

A comparison of genes that were present in one clade but not another revealed that clade A (A1 plus A2) and clade B primarily show differences in genes encoding proteins with functions in metabolic and environmental adaptations, and gene inactivations.

such as carbohydrate uptake and metabolism. Similarly, clades A1 and $\mathrm{A} 2$ primarily differed in genes associated with carbohydrate uptake and utilization, which indicates that access to a new source of carbon and energy is a major driver of E. faecium evolution. Analysis of the genes that are under selective pressure provided further support for this hypothesis, as genes encoding carbohydrate transport systems were among those found to be under positive selective pressure in clade A1. The E. faecium clades also differed in the presence of antibiotic-resistance genes; for example, genes conferring vancomycin and aminoglycoside resistance were absent from clade $B$ but present in most clade $\mathrm{A}$ isolates.

The authors estimate that clade A and clade $B$ diverged $~ 3,000$ years ago (possibly owing to changes in ecology associated with increased urbanization, animal domestication and improved hygiene practices), and clade $\mathrm{A}$ then further split into A1 (associated with hospitalacquired infections) and A2 (associated with animals and occasional human infections) 75 years ago, around the time that antibiotics were introduced in medicine and agriculture. In both cases, the split was associated with a switch in metabolic pathways to reflect the newly colonized niche and the acquisition of more antibiotic-resistance genes.

Rachel David strains show great variation (more than $5 \%$ ) in genome sequence. To investigate these changes and to better define the different $E$. faecium populations, the authors sequenced

\footnotetext{
ORIGINAL RESEARCH PAPER Lebreton, F., et al. Emergence of epidemic multidrug-resistant

Enterococcus faecium from animal and commensal strains. mBio 4, e00534-13 (2013)
} 